\title{
Compressive sensing-based channel estimation for SC-FDE system
}

\author{
Lu Si ${ }^{*}$ Xinle Yu, Hang Yin and Weizhang Xu
}

\begin{abstract}
The channel of wireless broadband communication system usually features time domain sparsity. The channel estimation of single carrier frequency domain equalization (SC-FDE) system was modeled as compressive sensing (CS)-based reconstruction of sparse signal. Matching pursuit algorithms based on greedy search were adopted to reconstruct channel information. Under the premise of reduced reference signal overhead, the system reliability can be effectively enhanced. This paper proposes a priori information aided scheme. By inserting the cyclic prefix of the first training sequence to a SC-FDE transmission frame, which is used as guard interval, the priori information including more accurate channel length and sparsity can be obtained with inter-block-interference (IBI)-free training sequence. The proposed scheme shows obvious superiority in slow fading channels. It effectively improves the reconstruction of probability, thereby improving the accuracy of channel estimation, meanwhile sacrificing less spectral efficiency.
\end{abstract}

Keywords: Compressive sensing, SC-FDE, OMP, SP, Priori information

\section{Introduction}

Channel state detection represents one key technology in modern wireless communication system. The reference signal-based channel estimation method has significant advantages in small error and low complexity, thus widely applied in modern wireless transmission systems. Different from the traditional channel estimation method which only considers the maximum delay in channel transmission, the compressive sensing (CS) theory-based method takes advantage of time domain sparsity of wireless broadband communication channel, and adopts parameterized estimation method to accomplish channel state detection through the estimation of position and gain of each transmission path, which can decrease the length of reference signal and improve the performance of channel estimation.

Traditional channel estimation algorithms such as least square (LS) estimation and minimum mean square error (MMSE) algorithm have better performance under multipath enrichment of wireless channels, but the performance will be greatly weakened in case of sparse wireless channels (with few multipaths). The sparsity will

\footnotetext{
* Correspondence: silu@cuc.edu.cn

Engineering Center of Digital Audio and Video, Communication University of China, Beijing, China
}

be more obvious when the transmission bandwidth is wide, multipath delay is big, and number of paths is limited. At this time, using conventional pilot frequency-based channel estimation will lead to high channel overhead, with estimation accuracy greatly reduced. Therefore, channel estimation of sparse channels using CS algorithm has become a new focus. The CS theory shows that if the signal is sparse, then high-dimensional signal can be converted to low-dimensional signal through sensing matrix, and then the original signal can be reconstructed with high probability from a small number of low-dimensional signals by solving the optimization problem [1-3]. Compared with the conventional pilot-based channel estimation method, CS-based method can greatly reduce pilot overhead and improve the performance while improving spectral efficiency. Bajwa et al. applied compressive sensing to channel estimation and proposed compressive channel sensing (CCS) [4]. The literature [5, 6] studied CS-based underwater acoustic channel estimation; literature $[7,8]$ applied CS to channel estimation of ultra wideband (UWB) communication systems; literature [9] studied TDS-OFDM transmission method based on the CS theory. The signal structure of TDS-OFDM and single carrier frequency domain equalization (SC-FDE) 
system is quite similar, which utilizes known training sequence in the time domain as data block guard interval.

Accurate channel state is obtained to eliminate the effects of channel multipath and Doppler on the transmitted signal by using channel equalization. The SC-FDE applies frequency domain equalization to single carrier systems, and adopts block transmission scheme. Similar to OFDM, SC-FDE has advantages of low complexity and strong anti-interference characteristics. Meanwhile, it has low peak-to-average power ratio (PAPR) and insensitivity to frequency deviation [10], which is particularly suitable for broadband communication uplink systems and spaceborne launch systems that are sensitive to the power, weight, and volume of transmitting equipment.

The SC-FDE system adopts unique words (UW) as guard interval and reference signal, and proposes a series of corresponding channel estimation algorithms. In order to avoid the interference of UW sequences from data blocks due to the multipath delay spread, literature [11] used dual UW (DUW) as guard interval to effectively improve the performance, but the channel overhead is high as each UW length is equivalent to the maximum multipath delay. In order to reduce the channel overhead, an iterative processing method was proposed. The total UW length is equivalent to the maximum multipath delay, but the slow convergence rate makes it impossible to acquire channel state quickly, so the method is not suitable for fast time varying channels. Targeting at the above problem, this paper proposed a CS-based channel estimation method for SC-FDE system, in which single UW is used as guard interval and reference signal. The conventional reconstruction algorithm is optimized, and an improved scheme based on priori information is proposed. The proposed scheme can improve the reconstruction of probability compared to the conventional one, thereby improving the accuracy of channel estimation and enhancing the reliability of the system effectively.

\section{Methodology}

\subsection{Compressive sensing-based SC-FDE}

The signal structure of the SC-FDE transmission system based on training sequence is shown in Fig. 1a. Where $C_{i}=\left[c_{i,}, c_{i, 1}, \cdots, c_{i,} P_{-1}\right]^{T}$ is a UW with a length $\mathrm{P}$. Therefore, for any $i$ and $j, c_{i}=c_{j}$ is established, $X_{i}=\left[x_{i, 0}\right.$, $\left.x_{i, 1}, \cdots, x_{i, N-1}\right]^{T}$ is payload data block with a length $N$. Let discrete time channel impulse response with the maximum multipath spread length $L$ be $H_{i}=\left[h_{i,}, h_{i}\right.$, $\left.{ }_{1}, \cdots, h_{i, L}\right]^{T}$, which satisfies $P \geq L$. Record $M=P$-L. The received signal obtained after channel transmission, time-frequency synchronization, and the multipath interference is shown in Fig. 1b, c.

The received signal $D_{i}=\left[d_{i, 0}, d_{i, 1}, \cdots, d_{i, P_{-}}\right]^{T}$ corresponding to the known training sequence can be expressed as:

$$
D_{i}=\Psi_{\mathrm{i}} H_{i}+N_{i}
$$

$N_{i}$ is additive white Gaussian noise and $\Psi_{\mathrm{i}}$ is:

$$
\Psi_{\mathrm{i}}=\left[\begin{array}{ccccc}
c_{i, 0} & x_{i, N-1} & x_{i, N-2} & \cdots & x_{i, N-L} \\
c_{i, 1} & c_{i, 0} & x_{i, N-1} & \cdots & x_{i, N-L+1} \\
c_{i, 2} & c_{i, 1} & c_{i, 0} & \cdots & x_{i, N-L+2} \\
\vdots & \vdots & \vdots & \ddots & \vdots \\
c_{i, L} & c_{i, L-1} & c_{i, L-2} & \cdots & c_{i, 0} \\
\vdots & \vdots & \vdots & \ddots & \vdots \\
c_{i, P-1} & c_{i, P-2} & c_{i, P-3} & \cdots & c_{i, P-L-1}
\end{array}\right]_{P \times(L+1)}
$$

It can be seen from the matrix $\Psi_{i}$ that the last $M$ samples of the received signal are not interfered by the unknown data, which constitute the inter-block-interference (IBI)-free region of the training sequence, to be recorded as $R_{i}=\left[d_{i, L}, d_{i, L+1}, \cdots, d_{i, P-}\right.$ $\left.{ }_{1}\right]^{T} . V_{i}$ is last $M$ samples of $N_{i}$, then:

$$
R_{i}=\Phi_{\mathrm{i}} H_{i}+V_{i}
$$

where $\Phi_{\mathrm{i}}$ is a Toeplitz array:

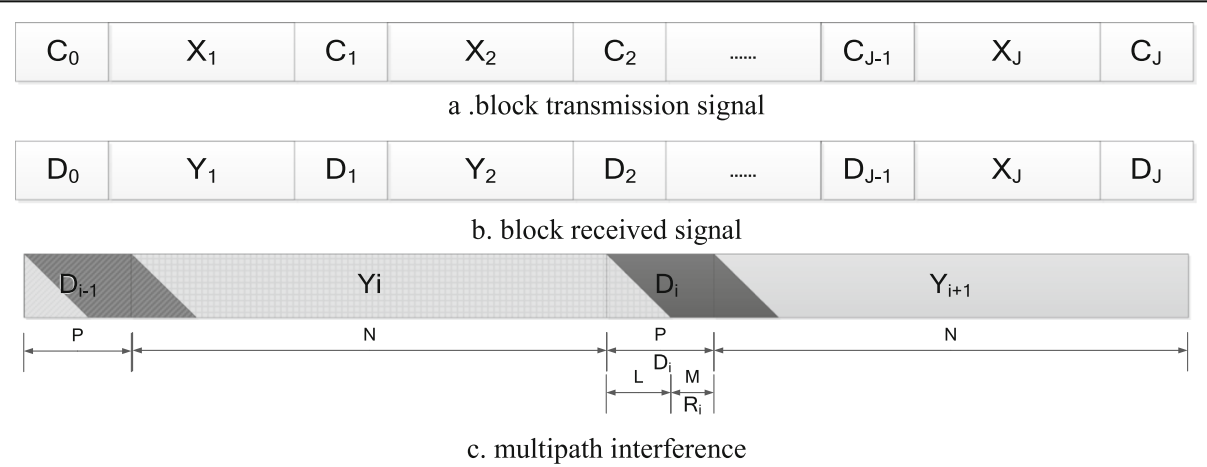

Fig. 1 The SC-FDE frame structure and multipath interference. a Block transmission signal. b Block received signal. c Multipath interference 


$$
\Phi_{\mathrm{i}}=\left[\begin{array}{ccccc}
c_{i, L} & c_{i, L-1} & c_{i, L-2} & \cdots & c_{i, 0} \\
c_{i, L+1} & c_{i, L} & c_{i, L-1} & \cdots & c_{i, 1} \\
\vdots & \vdots & \vdots & \ddots & \vdots \\
c_{i, P-1} & c_{i, P-2} & c_{i, P-3} & \cdots & c_{i, P-L-1}
\end{array}\right]_{M \times(L+1)}
$$

For SC-FDE of DUW, $M=L+1$, the matrix $\Phi_{i}$ is column full rank, then Eq. (3) can be used to estimate the channel state using conventional algorithms such as LS and MMSE. For broadband wireless channels, the channel state $H_{i}$ energy is mainly concentrated in a few elements and can be approximated as:

$$
H_{i}(\mathrm{n})=\sum_{k=0}^{K-1} \alpha_{i, k} \delta\left(n-\tau_{i, k}\right) \quad \mathrm{n} \in[0, \mathrm{~L}]
$$

The gain of the $k$ th path is $\alpha_{i, k}$, and the delay is $\tau_{i, k}$. As can be seen from the Formula (5), the element value of $H_{i}$ is $\alpha_{i, k}$ at $\tau_{i, k}$, the other element values are 0 , and there are $K$ non-zero elements. Record the path delay set as $\Omega_{i}=\left[\tau_{i, 0}, \tau_{i, 1}, \cdots, \tau_{i, K-1}\right]$, the path gain set as $A_{i}$ $=\left[\alpha_{i, 0}, \alpha_{i, 1}, \cdots, \alpha_{i, K-1}\right]$. Without loss of generality, suppose $0 \leq \tau_{i, 0} \leq \tau_{i, 1} \leq \ldots \leq \tau_{i, K-1} \leq \mathrm{L}-1$. In general, $K<<L$, that is, the channel has a high sparsity, so the channel estimation can be performed using CS algorithm. In CS algorithms, $R_{i}$ is referred to as measurement vector, $\Phi_{i}$ is a measurement matrix, $M$ is number of measurements, and $K$ is sparsity of $H_{i}$. The idea of compressive sensing is to turn the problem of finding $L$ unknowns $H_{i}$ into a problem of estimating $2 K$ unknowns $\Omega_{i}, A_{i}$, the key of which lies in correct estimation of $\Omega_{i}$. The reduced number of key unknowns enables effective improvement of performance in channel estimation. When $M<L+1$, the problem of solving $H_{i}$ in Formula (3) becomes a problem of solving underdetermined equation. $H_{i}$ is a sparse variable, then according to compressive sensing theory, the underdetermined equation can be solved by solving the following optimization problem to obtain the estimated channel parameter $H_{i}$ :

$$
\hat{H}_{i}=\underset{H_{i}}{\arg \min }\left\|H_{i}\right\|_{0} \text { s.t. }\left\|R_{i}-\Phi_{i} H_{i}\right\|_{2} \leq \varepsilon
$$

Where $\varepsilon$ is a non-negative constant related to noise. In recent years, compressive sensing theory has been developed to provide corresponding algorithms for solution of the above problems, including basis pursuit (BP) algorithm, greedy search-based matching pursuit (MP) algorithm, and relaxation convex optimization algorithm. Among them, MP is the most widely used reconstruction algorithm with the lowest complexity and the largest number of measurements.

Take the normalized random measurement matrix proposed in literature [12] as an example. Record it as $G_{i}=\Phi_{i}^{T} \Phi_{i}, G_{i}$ is a Hermitian matrix. When all elements in $\Phi_{i}$ are identically distributed random numbers and the columns are normalized (the sum of the squares of the column elements is 1 ), the diagonal elements of $G_{i}$ are always 1 , and the remaining elements are normally distributed random numbers with a mean of 0 and a variance of $1 / M$. Columns with index equal to $\Omega_{i}$ are taken from the matrix $G_{i}$ to constitute matrix $B$, rows with index equal to $\Omega_{i}$ are taken from matrix $B$ to constitute the sub-matrix $I+G_{i \Omega_{i}}$ ( $I$ is a unit matrix). The remaining rows of matrix $B$ make up a sub-matrix $G_{i \Omega_{i}^{\prime}}$. Taking the orthogonal matching pursuit (OMP) algorithm as an example, the condition [13] for correct choice of $\tau_{i, k}$ in each iteration by OMP is that the inequation (7) is established.

$$
\left\|\left(I+G_{i \Omega_{i}}\right) A_{i}\right\|_{\infty}>\left\|G_{i \Omega_{i}^{\prime}} A_{i}\right\|_{\infty}
$$

All the elemental and non-diagonal elements of $G_{i \Omega_{i}^{\prime}}$ are normally distributed random numbers with a mean value of 0 and a variance of $1 / M$. Therefore, for a larger $M$, the establishment probability of inequation (7) is higher. That is, the accurate rate in estimating $\Omega_{i}$ can be increased by increasing the measurements number $M$.

\subsection{Parameterized channel estimation based on priori information}

Reconstruction algorithm represents the development focus of compressive sensing theory. Among the existing greedy search-based algorithms, OMP and subspace pursuit (SP) are the two most widely used ones. However, like other matching pursuit algorithms, both algorithms demand signal sparsity and channel length as priori information. These two parameters are unknown in the receiving end of wireless communication, so the practicality is limited. Literature [9] proposed a PA-CoSaMP algorithm. Based on the good autocorrelation property of the training sequence, the priori information including rough estimation of channel length and sparse level are obtained. However, since the received training sequence are contaminated by the interference caused by the preceding data block, the estimated prior information need to be compensated. This paper proposed a priori information aided (PIA) scheme, which is suitable for most matching pursuit algorithms. Several consecutive data blocks are taken as a frame according to the time correlation of wireless channel. It is considered that the path delay of the channel within a frame remains unchanged and the path gain varies slightly. In each frame, a cyclic prefix of the first training sequence is inserted as guard interval to resist IBI. The length is taken as the maximum delay spread of the channel. Then the training sequence without IBI can be used to obtain the channel priori information, which 
lead to a higher reconstruction of probability. The frame structure for the proposed scheme is shown in Fig. 2.

As shown in Fig. 2, $C_{n, 0}$ consists of UW with a length of $P$ and its cyclic prefix, let $C_{n, 0}=$ $\left[c_{P-L_{\max }}, c_{\left.P-\operatorname{Lmax}+1, \cdots, c_{P-1}, c_{0}, c_{1}, \cdots, c_{P-1}\right]}^{T}\right.$, and $L_{\max }$ is equal to the length of maximum delay spread that may exist in the actual channel. Set $L_{\max }=\tau_{\max } \times f_{s}$, in which $\tau_{\max }$ is the maximum multipath delay and $f_{s}$ is the sampling rate. Take $J$ consecutive blocks of data as a frame, so for any $i, j \in[1, J]$, there is $c_{n, i}=c_{n, j}=\left[c_{0}, c_{1}, \cdots, c_{P-1}\right]^{T}$. The coherence time of the channel is assumed to be $T_{c}$, i.e., $T_{c}$ $=1 / f_{d}$, and $f_{d}$ is the Doppler frequency shift, which can be easily estimated at the receiving end [14]. When $J=$ $T_{c} /\left(T_{s}(P+N)\right)-1$, it is considered that the path delay of the channel remains unchanged and the path gain varies slightly within J consecutive data blocks.

The key to the PIA scheme lies in the estimation of channel length and sparsity. According to LS criterion, the last $\mathrm{P}$ samples of the known sequence $C_{n, 0}$ without interference from unknown data can be used to obtain the channel frequency domain response $\hat{H}_{n}$ of the $n$th frame, and $\hat{H}_{n}=\left[\hat{H}_{n, 0}, \hat{H}_{n, 1}, \cdots, \hat{H}_{n, P-1}\right]^{T}$. Let $h_{n}=\left[h_{n, 0}\right.$, $\left.h_{n, 1}, \cdots, h_{n, P_{-1}}\right]^{T}$ be the channel time domain impulse response of the nth frame, then $\hat{H}_{n}$ and $h_{n}$ will satisfy the relationship in (8):

$$
\hat{H}_{n}=W_{P} h_{n}+n_{p}
$$

Where $W_{P}$ is the Fourier transform matrix in the size of $P \times P, n_{p}$ is the white Gaussian noise vector of $P \times 1$, the variance is $\sigma_{\mathrm{n}}^{2}$. Owing to the unique inverse existence of $W_{B} h_{n}$ can be reduced to inverse Fourier transform of $\hat{H}_{n} \quad$ at the point $P$, i.e., $\hat{h}_{n}=W_{P}^{-1} \hat{H}_{n}$. Let $\hat{h}_{n}=$ $\left[\hat{h}_{n, 0}, \hat{h}_{n, 1}, \cdots, \hat{h}_{n, P-1}\right]^{T}$, then the estimate $\hat{S}_{n}$ of impulse response power in the nth frame can be calculated as $\hat{S}_{n}$ $=\left[\hat{S}_{n, 0}, \hat{S}_{n, 1}, \cdots, \hat{S}_{n, P-1}\right]^{T}=\left[\left|\hat{h}_{n, 0}\right|^{2},\left|\hat{h}_{n, 1}\right|^{2}, \cdots,\left|\hat{h}_{n, P-1}\right|^{2}\right]$. By averaging the power of $\hat{S}_{n}$ within two consecutive frames, the estimated power of each path $\hat{S}$ can be obtained, so $\hat{S}=\frac{1}{2}\left(\hat{S}_{n}+\hat{S}_{n+1}\right)$. $\hat{S}$ can be seen as a representation of the delay power spectrum which reflects statistical properties of the channel $[15,16]$. In this algorithm, $\hat{S}$ is defined as a time observation and corresponding time parameters can be extracted from it. Let $\hat{S}=\left[\hat{S}_{0}, \hat{S}_{1}, \cdots, \hat{S}_{P-1}\right]^{T}$, then the elements in $\hat{S}$ meet the definition in (9):

$$
\hat{S}_{l}=\frac{1}{2} \sum_{i=n}^{n+1}\left|\hat{h}_{i, l}\right|^{2}, l=0,1, \ldots, P-1
$$

The elements in the vector $\hat{S}$ are arranged in descending order to obtain vector $\hat{S}^{\prime}=\left[\hat{S}_{0}^{\prime}, \hat{S}_{1}^{\prime}, \cdots, \hat{S}_{P-G}^{\prime}, \hat{S}_{P-1}^{\prime}\right]^{T}$. The later $G$ elements of $\hat{S}^{\prime}$ are selected to represent the noise power. In simulation experiments, $G$ is selected according to different channel conditions. The latter $G$ elements are averaged to obtain average noise $N_{a v}$, which is subtracted from time observation $\hat{S}$ to obtain the vector, i.e., $\hat{S}^{\prime \prime}=\hat{S}-N_{a v} . \hat{S}^{\prime \prime}$ is searched according to (10) criterion to obtain the index:

$$
\hat{\Omega}_{n}=\left\{l \mid \hat{S}_{l}^{\prime \prime}>\gamma, l=0,1, \ldots, P-1\right\}
$$

The elements in the index set $\hat{\Omega}_{n}$ obtained according to this criterion can be regarded as an estimate of the multipath delay position for the nth frame. The threshold will be determined by Formula (11) [17]:

$$
\gamma=\max \left\{\hat{S}_{\max }^{*} 10^{-\left(\gamma_{1} / 10\right)}, \hat{S}_{\min }^{*} 10^{\gamma_{2} / 10}\right\}
$$

Where, $\hat{S}_{\max }^{\prime \prime}$ and $\hat{S}_{\min }^{\prime \prime}$ respectively represent the maximum and minimum values in $\hat{S}^{\prime \prime}, \gamma_{1}, \gamma_{2}$ are a representation of the energy $\hat{S}^{\prime \prime}$, which are determined by simulation experiments according to different systems in specific calculations. Suppose $\hat{L}=\max \left\{\hat{\Omega}_{n}\right\}$ as the value of estimated channel length, and $\hat{K}=\left\|\hat{\Omega}_{n}\right\|_{0}$ as the channel sparsity. According to the estimated value $\hat{L}$, the position of the IBI-free region in the received training sequence is determined. The input parameters of the reconstruction algorithms are initialized. The measurement matrix $\Phi_{i}$ at each moment is obtained from the channel length $\hat{L}$, as shown in Eq. (12).

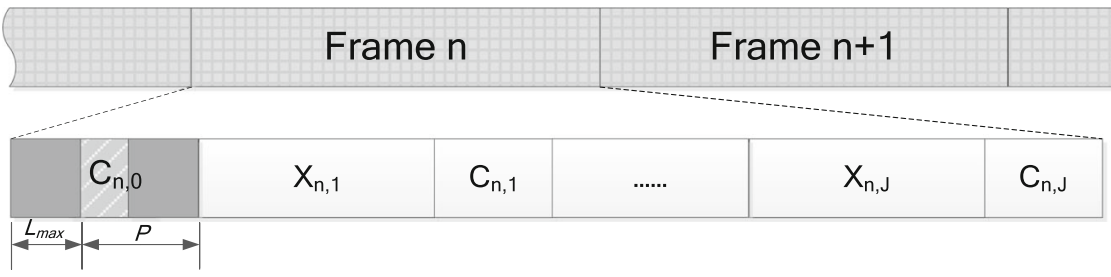

Fig. 2 The frame structure of SC-FDE for PIA scheme 


$$
\Phi_{\mathrm{i}}=\left[\begin{array}{ccccc}
c_{i, \hat{L}} & c_{i, \hat{L}-1} & c_{i, \hat{L}-2} & \cdots & c_{i, 0} \\
c_{i, \hat{L}+1} & c_{i, \hat{L}} & c_{i, \hat{L}-1} & \cdots & c_{i, 1} \\
\vdots & \vdots & \vdots & \ddots & \vdots \\
c_{i, P-1} & c_{i, P-2} & c_{i, P-3} & \cdots & c_{i, P-\hat{L}-1}
\end{array}\right]_{M \times(\hat{L}+1)}
$$

The measurement vector is $R_{i}=$ $\left[d_{i, \hat{L}}, d_{i, \hat{L}+1}, \cdots, d_{i, P-1}\right]^{T}$, and the number of measurements is $M=P-\hat{L}$. The subsequent steps are consistent with those of classic MP algorithms and will not be elaborated here. The OMP and SP algorithms based on prior information are referred to as PIA-OMP and PIA-SP respectively in what follows.

Regarding to the proposed PIA scheme, $\hat{H}_{n}$ can be efficiently calculated by $P$-point FFT to realize the LS estimation, and $\hat{h}_{n}$ can be calculated by $P$-point IFFT. Same as the conventional methods, the main computational burden of the PIA scheme comes from the MP algorithm.

\section{Simulation results and discussion}

This paper sets up a SC-FDE system simulation platform and sets the symbol rate at $f_{s}=8 K S p s$. The main parameters of the sending end are shown in Table 1 . The wide sense stationary uncorrelated scattering (WSSUS) channel model is adopted in this simulation. The maximum multipath delay is set as $\tau_{\max }=5 \mathrm{~ms}$ based on the ionospheric channel characteristics. The channel sparsity $K$ $=2$, the Doppler spread $f_{d}=1 \mathrm{~Hz}$, and the gain is independent for different propagation paths. Assume that the system is perfectly synchronized.

Let the length of UW $P=64$ and the length of data block $N=512$, and every $J(J=12)$ data blocks are selected as a frame. Where UW is in Chu sequence with constant envelope characteristics [18]. For different estimation methods, the reference signal length and spectral efficiency are shown in Table 2. For LS, MMSE, OMP, and SP schemes, spectral efficiency is $\eta=\frac{N}{N+P}$. In particular, spectral efficiency for PIA-OMP and PIA-SP is $\eta$ $=\frac{J N}{J(N+P)+\left(L_{\max }+P\right)}$. From the table, it can be observed that PIA schemes sacrifice less spectral efficiency. But when $J$ gets smaller, the decrease in spectral efficiency cannot be ignored. Therefore, the proposed scheme is not suitable for fast fading channels. However, its application in slow fading channels shows obvious superiority.

Table 1 Modulation and coding parameters

\begin{tabular}{ll}
\hline Parameter & Value \\
\hline Modulation & QPSK \\
Coding & Convolution code \\
Code rate & 0.5 \\
\hline
\end{tabular}

Table 2 The reference signal and spectral efficiency comparison

\begin{tabular}{llll}
\hline Channel estimation scheme & LS/MMSE & OMP/SP & PIA-OMP/PIA-SP \\
\hline Reference signal & DUW & UW & UW \\
Data block length (N) & 512 & 512 & 512 \\
Reference signal length (P) & 128 & 64 & 64 \\
Spectral efficiency $(\eta)$ & $80 \%$ & $88.9 \%$ & $87.6 \%$ \\
\hline
\end{tabular}

What needs illustration is that conventional OMP and SP assumed that the channel sparsity is known, and the channel length takes the value $L_{\max }=\tau_{\max } \times$ $f_{s}$. Figure 3 shows the power of time domain impulse response of the channel obtained with the proposed scheme with $\mathrm{SNR}=10 \mathrm{~dB}$. Accurate two-path delay information can be obtained from the figure. The MSE performance comparison between the proposed schemes and its conventional counterparts is given in Fig. 4. For LS and MMSE algorithms, dual UW is used as guard interval and reference signal. It can be seen from the figure that, under the premise of reducing the reference signal length by half, OMP and $\mathrm{SP}$ result in a SNR gain of more than $5 \mathrm{~dB}$ when MSE $=10^{-2}$ compared with conventional MMSE based on DUW; the proposed PIA-SP result in a SNR gain of about $4 \mathrm{~dB}$ when $\mathrm{MSE}=10^{-2} \mathrm{com}-$ pared with SP, the proposed PIA-OMP result in a SNR gain of about $2.5 \mathrm{~dB}$ when $\mathrm{MSE}=10^{-3}$ compared with OMP. This is because a longer measurement vector is utilized, and the reconstruction of probability is improved. As SNR goes up, the performance of PIA schemes approaches the theoretical CRLB lower bound [19]. Figure 5 shows the bit-error-rate (BER) curves for different methods. It can be observed that OMP and SP perform closely to MMSE, but PIA-OMP and PIA-SP outperforms

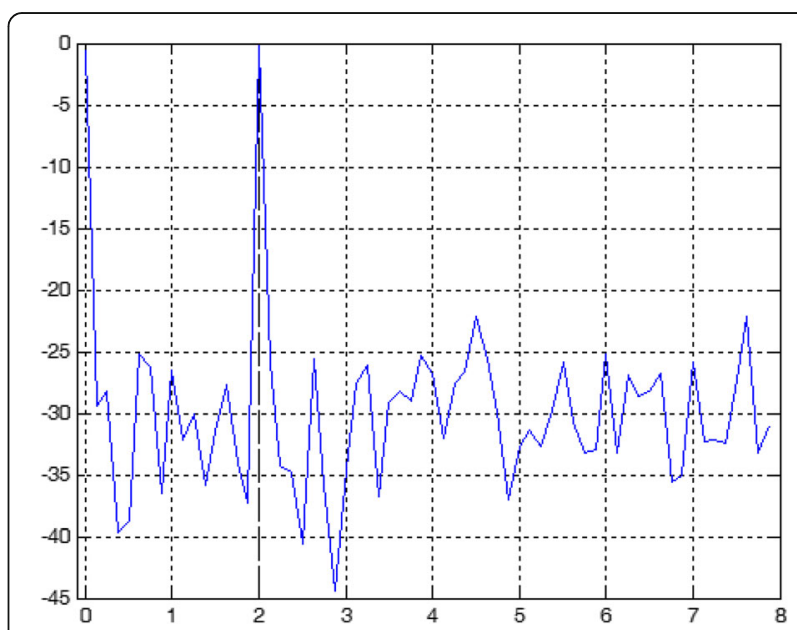

Fig. 3 The power of time domain pulse response with $S N R=10 \mathrm{~dB}$. In Fig. 3, X-axis is the power of time domain pulse response $(\mathrm{dB})$ and $Y$-axis is time (ms) 


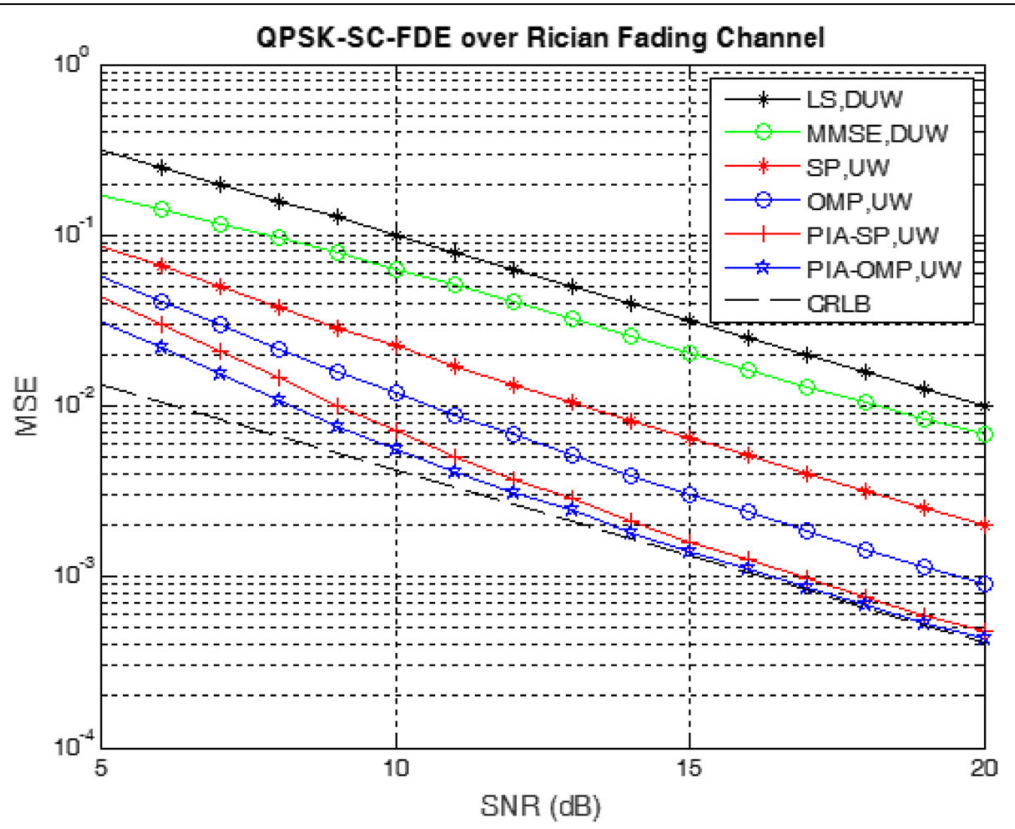

Fig. 4 The MSE performance comparison between the proposed channel estimation scheme and its conventional counterparts with $P=64$

the conventional MMSE by more than $1 \mathrm{~dB}$ at the target BER of $10^{-4}$.

In addition, the simulation tests the performance of CS-based schemes when adopting different number of measurements $M$. Figures 6 and 7 show the signal reconstruction performance and BER performance when varying number of measurements is utilized with SNR =
$15 \mathrm{~dB}$. As described in the previous section, for OMP and SP, $M$ is equal to the value of the horizontal axis, and the length of reference signal is $P=M+L_{\max }$; for PIA-OMP and PIA-SP, the actual number of measurements $M^{\prime}$ is always bigger than the horizontal value, and $M^{\prime}=P-\hat{L}=M+L_{\max }-\hat{L}$. It is clear that the proposed scheme performs better than others when the

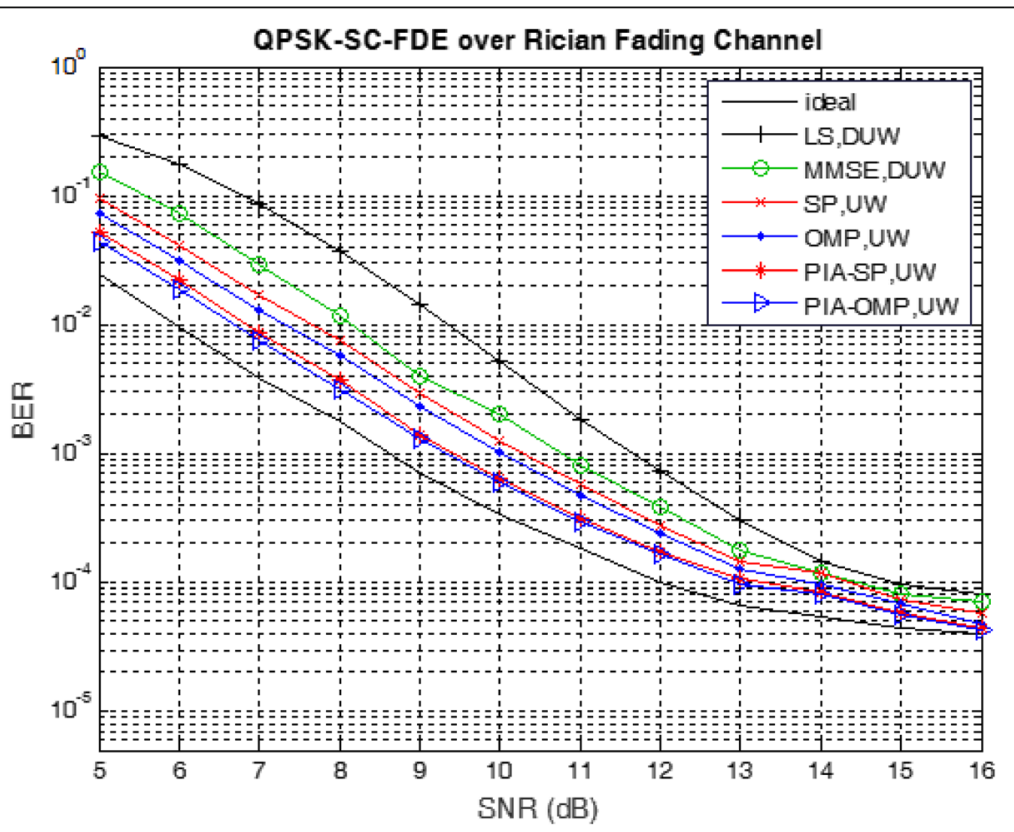

Fig. 5 The BER performance comparison between the proposed channel estimation scheme and its conventional counterparts with $P=64$ 


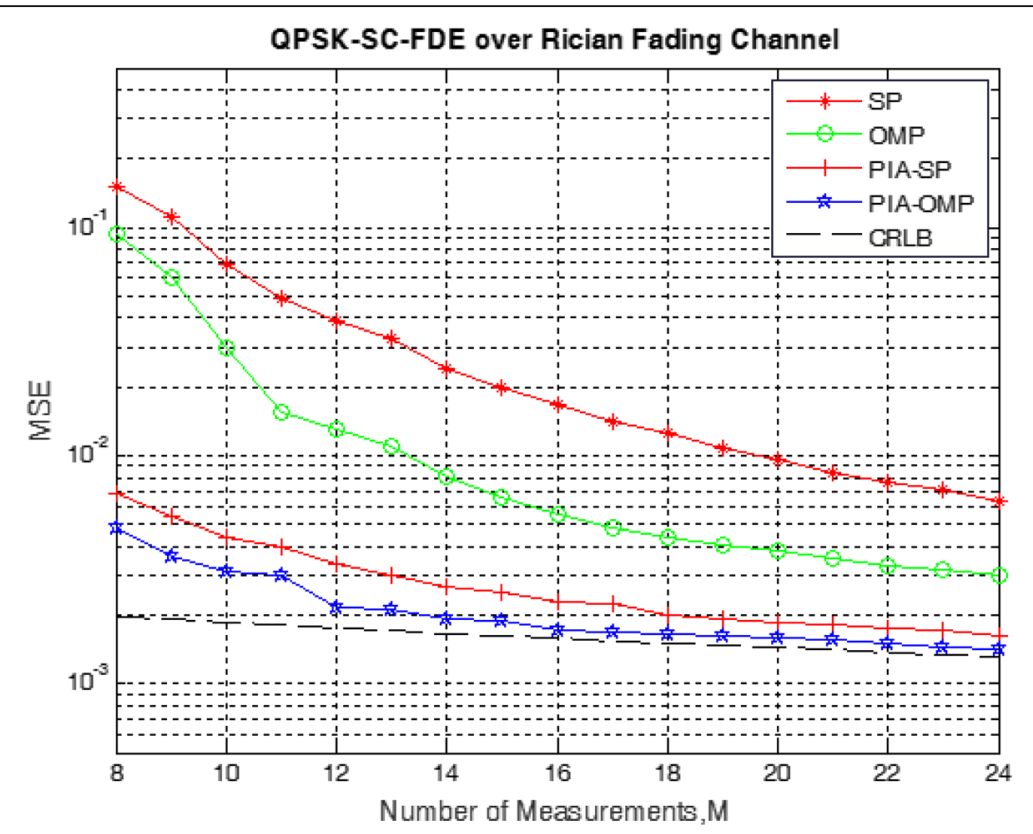

Fig. 6 Signal reconstruction performance when varying number of measurements is utilized with $S N R=15 \mathrm{~dB}$

number of measurements is small, e.g., $M<7 K$. When $M$ goes up, the BER performance of proposed schemes approach the ideal estimation.

\section{Conclusion}

Taking advantage of the sparsity of wireless broadband channels, this paper models the channel estimation of SC-FDE system as a CS-based sparse signal recon struction problem, and uses greedy search-based matching pursuit algorithm to reconstruct channel information. The CS-based methods can improve the performance of channel estimation effectively, meanwhile reducing the reference signal overhead. This paper proposes a priori information aided scheme. By inserting a cyclic prefix of the first training sequence to a SC-FDE transmission frame, which is used as guard interval, the

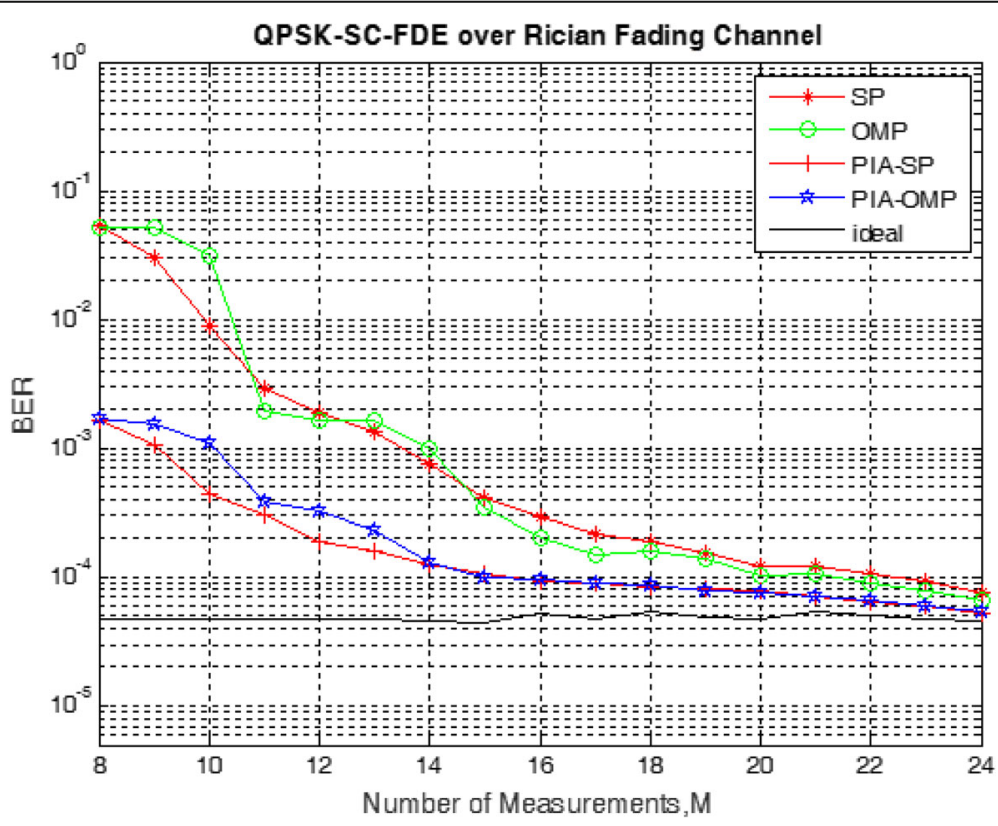

Fig. 7 The BER performance when varying number of measurements is utilized with $S N R=15 \mathrm{~dB}$ 
priori information including more accurate channel length and sparsity can be obtained with IBI-free training sequence. The proposed scheme shows obvious superiority in slow fading channels. Compared with the conventional methods, the PIA scheme effectively improves the reconstruction of probability, while sacrificing less spectral efficiency. What is more, the proposed scheme is more universal due to the acquisition of priori information.

\section{Abbreviations}

BER: Bit-error-rate; CS: Compressive sensing; IBI: Inter-block-interference; OMP: Orthogonal matching pursuit; PIA: Priori information aided; SC-

FDE: Single carrier frequency domain equalization; SP: Subspace pursuit

\section{Acknowledgements}

The research presented in this paper was supported by Communication University of China, Beijing, China.

\section{Funding}

This work is supported by the Sponsor of 2015BAK05B01, National Key Technology Support Program.

\section{Availability of data and materials}

Not applicable.

\section{Authors' contributions}

$L S$ is the main writer of this paper. She proposed the main idea, completed the simulation, and analyzed the result. XY and WX provides mathematical and statistical guidance for this paper. HY gave some important suggestions for the simulation. All authors read and approved the final manuscript.

\section{Competing interests}

The authors declare that they have no competing interests.

\section{Publisher's Note}

Springer Nature remains neutral with regard to jurisdictional claims in published maps and institutional affiliations.

Received: 3 July 2018 Accepted: 21 December 2018

Published online: 21 January 2019

\section{References}

1. D.L. Donoho, Compressed sensing. IEEE Trans. Inf. Theory 52(4), 1289-1306 (2006)

2. R.G. Baraniuk, Compressive sensing. IEEE Signal Process. Mag. 24(4), 118-121 (2007)

3. E.J. Candès, M.B. Wakin, An introduction to compressive sampling. IEEE Signal Process. Mag. 25(2), 21-30 (2008)

4. W.U. Bajwa, J. Haupt, A.M. Sayeed, et al., Compressed channel sensing: a new approach to estimating sparse multipath channels. Proc. IEEE 98(6), 1058-1076 (2010)

5. C.R. Berger, S. Zhou, J.C. Preisig, et al., Sparse channel estimation for multicarrier underwater acoustic communication: from subspace methods to compressed sensing. IEEE Trans. Signal Process. 58(3), 1708-1721 (2010)

6. C. Qi, X. Wang, L. Wu, Underwater acoustic channel estimation based on sparse recovery algorithms. IET Signal Process 5(8), 739-747 (2011)

7. J.L. Paredes, G.R. Arce, Z. Wang, Ultra-wideband compressed sensing: channel estimation. IEEE J. Sel. Top. Sign. Proces. 1(3), 383-395 (2007)

8. A.H. Muqaibel, M.T. Alkhodary, Practical application of compressive sensing to ultra-wideband channels. IET Commun. 6(16), 2534-2542 (2012)

9. L. Dai, Z. Wang, Z. Yang, Compressive sensing based time domain synchronous OFDM transmission for vehicular communications. IEEE J. Sel. Areas Commun. 31(9), 460-469 (2013)

10. N. Benvenuto, R. Dinis, D. Falconer, S. Tomasin, Single carrier modulation with nonlinear frequency domain equalization: An idea whose time has ComeAgain. Proc. IEEE 98(1), 69-96 (2010)
11. Y. Zeng, T.S. Ng, Pilot cyclic prefixed single carrier communication: channel estimation and equalization. IEEE Signal Process Lett. 12(1), 56-59 (2005)

12. J.A. Tropp, A.C. Gilbert, Signal recovery from random measurements via orthogonal matching pursuit. IEEE Trans. Inf. Theory 53(12), 4655-4666 (2007)

13. J.A. Tropp, Greed is good: algorithmic results for sparse approximation. IEEE Trans. Inf. Theory 50(10), 2231-2242 (2004)

14. J. Cai, W. Song, Z. Li, Doppler spread estimation for mobile OFDM systems in Rayleigh fading channels. IEEE Trans. Consum. Electron. 49(4), 973-977 (2003)

15. J.J. Van de Beek, M. Sandell, P.O. Borjesson, ML estimation of time and frequency offset in OFDM systems. IEEE Trans. Signal Process. 45(7), 18001805 (1997)

16. Y. Li, L.J. Cimini, N.R. Sollenberger, Robust channel estimation for OFDM systems with rapid dispersive fading channels. IEEE Trans. Commun. 46(7), 902-915 (1998)

17. B. Yang, K.B. Letaief, R.S. Cheng, Z. Cao, Timing recovery for OFDM transmission. IEEE J. Sel. Areas Commun. 18(11), 2278-2291 (2000)

18. D.C. Chu, Polyphase codes with good periodic correlation properties. IEEE Trans. Inf. Theory 18(4), 531-532 (1972)

19. B. Yang, K. Letaief, R. Cheng, Z. Cao, Channel estimation for OFDM transmission in multipath fading channels based on parametric channel modeling. IEEE Trans. Commun. 49(3), 467-479 (2001)

\section{Submit your manuscript to a SpringerOpen ${ }^{\circ}$ journal and benefit from:}

- Convenient online submission

- Rigorous peer review

- Open access: articles freely available online

- High visibility within the field

- Retaining the copyright to your article

Submit your next manuscript at $\boldsymbol{\nabla}$ springeropen.com 